\title{
Trait selection during food web assembly: the roles of interactions and temperature
}

\author{
Isabelle Gounand $^{1,2,3,4,5} \cdot$ Sonia Kéfi ${ }^{1} \cdot$ Nicolas Mouquet $^{1} \cdot$ Dominique Gravel $^{2,3}$
}

Received: 7 December 2015 / Accepted: 23 March 2016/Published online: 11 April 2016

(C) Springer Science+Business Media Dordrecht 2016

\begin{abstract}
Understanding the processes driving community assembly is a central theme in ecology, yet this topic is marginally studied in food webs. Bioenergetic models have been instrumental in the development of food web theory, using allometric relationships with body mass, temperature, and explicit energy flows. However, despite their popularity, little is known about the constraints they impose on assembly dynamics. In this study, we build on classical consumer-resource theory to analyze the implications of the assembly process on trait selection in food webs. Using bioenergetic models, we investigate the selective pressure on body mass and conversion efficiency and its dependence on trophic structure and temperature. We find that the selection exerted by exploitative competition is highly sensitive to how the energy fluxes are modeled. However, the addition of a trophic level consistently
\end{abstract}

Electronic supplementary material The online version of this article (doi:10.1007/s12080-016-0299-7) contains supplementary material, which is available to authorized users.

Isabelle Gounand

isabelle.gounand@eawag.ch

Sonia Kéfi

sonia.kefi@univ-montp2.fr

Nicolas Mouquet

nicolas.mouquet@cnrs.fr

Dominique Gravel

dominique.gravel@usherbrooke.ca

Institut des Sciences de l'Evolution, Université de Montpellier, CNRS, IRD, EPHE, CC 065, Place Eugène Bataillon,

34095 Montpellier Cedex 05, France selects for smaller body masses of primary producers. An increase in temperature triggers important cascading changes in food webs via a reduction of producer biomass, which is detrimental to herbivore persistence. This affects the structure of trait distributions, which in turn strengthens the exploitative competition and the selective pressure on traits. Our results suggest that greater attention should be devoted to the effects of food web assembly on trait selection to understand the diversity and the functioning of real food webs, as well as their possible response to ongoing global changes.

Keywords Community assembly $\cdot$ Consumer-resource interactions $\cdot$ Bioenergetic model $\cdot$ Temperature $\cdot$ Size spectrum $\cdot$ Body mass $\cdot$ Metabolic theory of ecology

2 Département de Biologie, Chimie et Géographie, Université du Québec à Rimouski, 300 Allée des Ursulines, Québec, QC G5L 3A1, Canada

3 Quebec Center for Biodiversity Science, Quebec, QC, Canada

4 Department of Evolutionary Biology and Environmental Studies, University of Zurich, Winterthurerstrasse 190, CH-8057 Zürich, Switzerland

5 Department of Aquatic Ecology, Eawag, Swiss Federal Institute of Aquatic Science and Technology, Überlandstrasse 133, CH-8600 Dübendorf, Switzerland 


\section{Introduction}

Food webs result from an assembly process during which species continuously arrive into the ecosystem. Newcomers interact with the resident species and can either go extinct or persist, possibly excluding or coexisting with the resident species. During this selection process, species composition changes gradually, thereby shaping the trait distribution of the whole food web. We hereafter refer to this process as "trait selection." Consumer-resource theory provides clear predictions on simple interaction outcomes (Tilman 1982; Chesson and Kuang 2008), but it has, so far, not been integrated in the context of sequential assembly of highly diverse food webs. Most community assembly studies have focused on how the order of species arrival may produce alternative stable communities (Luh and Pimm 1993; Law and Morton 1993, 1996; Lockwood et al. 1997; Fukami 2005) and on how local diversity drives resilience and resistance to invasions (Post and Pimm 1983; Case 1990, 1991; Law and Morton 1996; Morton and Law 1997; Capitán et al. 2011). Here, we connect recent food web developments to community assembly research to understand the mechanisms driving food web development.

Consumer-resource theory establishes the conditions for species coexistence or competitive exclusion, when a few species are interacting (Tilman 1982; Chase and Leibold 2003; Murdoch et al. 2003). Coexistence within a trophic level is mainly constrained by exploitative competition (i.e., competition for resources) and apparent competition (i.e., competition that emerges from sharing a predator), which involve the relative efficiency of species to acquire resources and to withstand predation (Chesson and Kuang 2008). Experiments and theoretical studies have established that under exploitative competition, species with identical resources could not coexist indefinitely (so-called competitive exclusion principle of Gause 1934; Hardin 1960). The $\mathrm{R}^{*}$ rule states that the species whose traits allow to reduce the equilibrium density of the resource to the lowest level will exclude all of its competitors (Tilman 1977, 1980; Tilman et al. 1981; Miller et al. 2005). When species share a predator, theory predicts that the one that can sustain the greatest density of predators will win the apparent competition (Holt et al. 1994, 2001; Holt and Lawton 1994; Chase et al. 2002; Chesson and Kuang 2008). Thus, consumerresource theory provides an extensive understanding of the dynamics for a specific trophic level and, to a certain extent, of its resources and predators (Schmitz et al. 2000; Thébault and Loreau 2003, 2006; Schreiber and Rittenhouse 2004).

The consequences of exploitative and apparent competitions are clear in simple communities including a few species only (Chase and Leibold 2003; Murdoch et al. 2003). However, a general understanding of coexistence mechanisms responsible for the assembly of food webs, in which complex indirect interactions operate simultaneously, remains to be achieved. While many studies have analyzed the disassembly of food webs through secondary extinctions (Solé and Montoya 2001; Dunne et al. 2002; Gross and Cardinale 2005; Montoya et al. 2006; Dunne and Williams 2009; Fowler 2010), we know little about food web assembly (Bascompte and Stouffer 2009). Until now, assembly research has mainly investigated simple systems, such as plant communities (Götzenberger et al. 2012; Martin and Wilsey 2012) or experimental protist communities with few species (Louette and De Meester 2007; Olito and Fukami 2009). We expect the assembly process within a single trophic level to progressively select traits improving resource use as an outcome of exploitative competition. When a higher trophic level is added, the assembly process should also select traits improving prey resistance as an outcome of predation pressure. This has still to be demonstrated and tested in the context of multitrophic food webs in which multiple interactions occur simultaneously.

Yodzis and Innes (1992) took an important step toward the mechanistic understanding of consumer-resource dynamics by setting the bases of bioenergetic models. The formalism of the metabolic theory of ecology (Gillooly et al. 2001; Brown et al. 2004) facilitated the parameterization of complex food web models, reducing drastically the number of estimated parameters (e.g., Brose et al. 2006b, 2012; Petchey et al. 2008; Berlow et al. 2009). Body mass determines inflow (consumption) and outflow (mortality) rates, and conversion efficiency the proportion of inflow that a species is able to convert into new biomass. Such parameterized models have been studied to address the stability of consumer-resource systems (Yodzis and Innes 1992; Vasseur and McCann 2005). However, the implications of body mass constraints on coexistence have not been elucidated so far in speciesrich food webs. During the assembly process, successive colonization events should shape the distribution of body masses in the food web. Given that body mass is in many cases related to trophic levels (Cohen et al. 2003; Riede et al. 2011), this might have important implications for the structure of food webs.

Furthermore, biological rates are known to strongly vary with temperature (e.g., Rall et al. 2012), and bioenergetic models allow integrating this dependence (Vasseur and McCann 2005). Such models allow investigating how changes in temperature affect species interactions and trophic flows in food webs (Vasseur and McCann 2005; Gilbert et al. 2014). The construction of a food web through time is likely to be conditioned by metabolism and, hence, by temperature. How this may affect trait selection is an important issue to understand food web response to climate change.

In this study, we aim at understanding trait selection over the course of food web assembly, in the light of consumerresource theory. We develop an assembly model in which we implement metabolic constraints in the same manner as other bioenergetic models (e.g., Brose et al. 2006b; Berlow et al. 
2009). The model is used to follow the changes in trait distributions (i.e., body mass and conversion efficiency) during the assembly of food webs having different levels of trophic complexity (with and without herbivores) and at different temperatures. We investigate the mechanisms of trait selection through three more specific questions introduced above: Q1-How does selection act on species traits during assembly of competitive communities? Q2-How does the addition of a trophic level modify this trait selection? Q3-How does temperature affect trait selection via species metabolism?

\section{Methods}

For each of our three questions, we first study analytically small modules of interacting species, which reproduce the case of a species attempting to colonize an ecosystem at equilibrium (Fig. 1a-c). We then investigate whether the predictions on trait selection obtained from this analysis hold or not in simulated assemblies of multi-species food webs (Fig. 1d). To answer Q1, we focus on communities composed of a single trophic level (i.e., primary producers exploiting limiting nutrients, Fig. 1a). We compare the results of four slightly different models to assess the sensitivity of trait selection to model formulation and hypotheses. We then add one trophic level (i.e., herbivores, Fig. 1b, c) and compare trait distributions at the end of the assembly process in the presence or absence of herbivores to answer Q2. To answer Q3, we

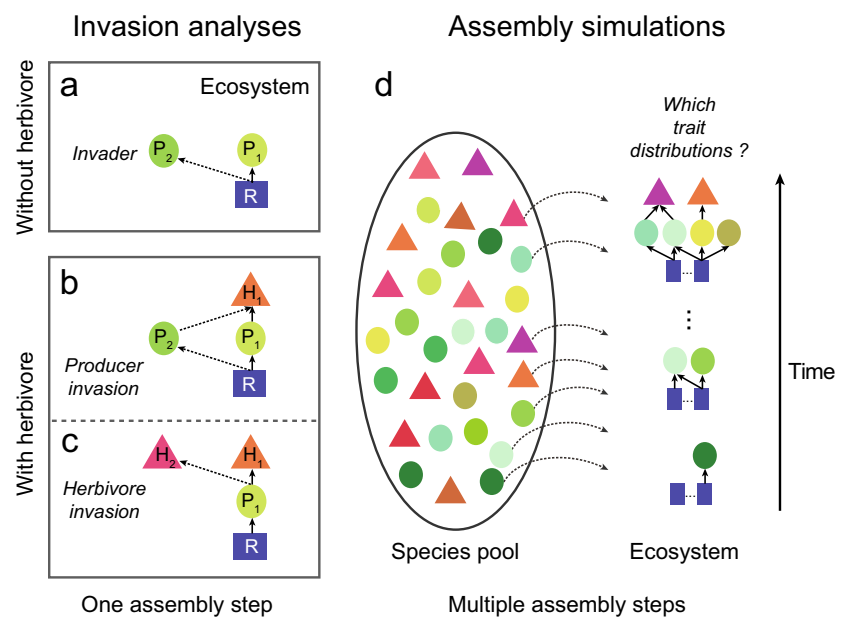

Fig. 1 Schematic representation of the methodology. We first performed invasion analyses of small modules (left panels) and then simulations of food web assembly (right panel). We analytically investigated competition between local residents and invaders during a hypothetical assembly step, when an herbivore is a absent or $\mathbf{b}$, $\mathbf{c}$ present in the food web. Invaders are either $\mathbf{a}, \mathbf{b}$ primary producers or $\mathbf{c}$ herbivores. We then simulated assemblies with multiple successive invasions (d). Rectangles, circles, and triangles refer respectively to resources, primary producers, and herbivores. We studied how these successive invasions affect the resulting trait distributions in the food web implement temperature dependence of biological rates and compare trait distributions of food webs assembled at two contrasting temperatures.

\section{Q1 Trait selection on a single trophic level}

In this section, we describe the four model variants studied to assess trait selection sensitivity to model assumptions, starting with a classical consumer-resource model (model 1), followed by the bioenergetic model (model 2) and its variants (models 3 and 4 ). We then explain the analytical and simulation procedures used to analyze trait selection.

Model 1: for the investigation of Q1, we consider a community made of a single trophic level of primary producers, $P_{j}$, feeding on inorganic resources $R_{i}$ (Fig. 1a). As a reference, we consider a classical consumer-resource model (as in DeAngelis and Mulholland 1989), hereafter called "Model 1". The dynamics are described by the following ordinary differential equations:

$\frac{d R_{i}}{d t}=I-$ out $_{R} R_{i}-\sum_{j} a_{j} R_{i} P_{j}$

$\frac{d P_{j}}{d t}=\varepsilon_{j} \sum_{i} a_{j} R_{i} P_{j}-m_{j} P_{j}$

The inorganic resource $R_{i}$ is supplied by external inputs $I$ (e.g., rainfall) and looses nutrients at rate out $_{R}$ (e.g., leaching). The primary producer $j$ uptakes resources at a rate $a_{j}$ (i.e., linear functional response). $P_{j}$ converts a proportion $\varepsilon_{j}$ of what it consumes into new biomass and looses biomass at a rate $m_{j}$ (e.g., mortality). The subscripts $i$ and $j$ refer to the identity of resources and species in multi-species simulations. Table 1 summarizes parameter and variable symbols and their interpretation and units.

Bioenergetic models We integrate metabolic constraints on bioenergetic flows in model 2 to study how body mass might be selected along the assembly. We replaced the uptake and mortality rates $a_{j}$ and $m_{j}$ of Eqs. [1] and [2] in model 1 by their bioenergetic equivalents: the per capita uptake rate $Y_{j}$ and the metabolic rate $X_{j}$. Both are defined by allometric relationships with specific body mass $M_{j}$ and temperature $T$ based on empirical data (Brown et al. 2004; Vasseur and McCann 2005) as follows:

$Y_{j}=a_{y_{\left(T_{0}\right)}} M_{j}^{-0.25} e^{\frac{E\left(T-T_{0}\right)}{k T T_{0}}}$

$X_{j}=a_{x_{\left(T_{0}\right)}} M_{j}^{-0.25} e^{\frac{E\left(T-T_{0}\right)}{k T T_{0}}}$

Biological rates display an exponential decrease with body mass, $M$. The temperature-dependent factor is derived from Arrhenius law (describing the increase of chemical reaction 
Table 1 Symbols and parameter values used in simulations

\begin{tabular}{|c|c|c|c|}
\hline Symbol & Description & Dimensions & Values \\
\hline$R, P, H$ & Densities of the compartments: inorganic resource, producer, herbivore & M. $L^{-2}$ & - \\
\hline$I$ & Input of inorganic nutrient & $M \cdot L^{-2} \cdot t^{-1}$ & 1 \\
\hline out $_{i}$ & Output rate of species or resource $i$ & $t^{-1}$ & 0.1 \\
\hline$M_{i}$ & Specific body mass of species $i$ & $M$ & $\log (\mathrm{M}) \in \mathcal{U}\left[10^{-8}, 10^{8}\right]$ \\
\hline$T$ & Temperature & $\Theta$ & $\left\{0,20,40^{\circ} \mathrm{C}\right\}$ used in ${ }^{\circ}$ Kelvin \\
\hline$T_{0}$ & Reference temperature & $\Theta$ & $20^{\circ} \mathrm{C}$ \\
\hline E & Activation energy & $M \cdot L^{2} \cdot t^{-2}$ & $0.62 \mathrm{eV}$ (Gillooly et al. 2001) \\
\hline$k$ & Constant of Boltzmann & $M \cdot L^{2} \cdot t^{-2} \cdot \Theta^{-1}$ & $8.61 \times 10^{-5} \mathrm{eV} \cdot \mathrm{K}^{-1}$ \\
\hline$\varepsilon_{i}$ & Conversion efficiency of species $i$ & dimensionless & $\mathcal{U}[0,1]$ \\
\hline$Y_{i}$ & Maximum per capita uptake rate of species $i$ in models 2 to 4 & $t^{-1}$ & \\
\hline$X_{i}$ & Metabolic rate of species $i$ in models 2 to 4 & $t^{-1}$ & \\
\hline$a_{x_{i}}$ & $\begin{array}{l}\text { Allometric constant for the metabolic rate of species } i \text { measured at temperature } T_{0} \text {. } \\
\text { The same constant is used for all species of the same trophic level }(\mathrm{P} \text { or H). }\end{array}$ & $t^{-1} \cdot M^{1 / 4}$ & $\begin{array}{l}\text { P: } 0.138 \\
\text { H: } 0.314 \text { invertebrates) } \\
\text { (Brose } \text { et al. 2006b) }\end{array}$ \\
\hline$a_{y\left(T_{0}\right)}$ & $\begin{array}{l}\text { Allometric constant for the uptake rate of species } i \text { measured at temperature } T_{0} \text {. } \\
\text { The same constant is used for all species of the same trophic level ( } \mathrm{P} \text { or H). }\end{array}$ & $t^{-1} \cdot M^{1 / 4} \cdot\left(M \cdot L^{-2}\right)^{-1}$ & $\begin{array}{l}\text { P: } 1 \\
\text { H: } 2.512 \text { (invertebrates) } \\
\text { (Brose } \text { et al. 2006b) }\end{array}$ \\
\hline$a_{i}$ & Maximum per capita uptake rate of species $i$ in model 1 & $t^{-1} \cdot\left(M \cdot L^{-2}\right)^{-1}$ & $\mathcal{U}[0,1]$ \\
\hline$m_{i}$ & Mortality rate of species $i$ in model 1 & $t^{-1}$ & $\mathcal{U}[0.01,1]$ \\
\hline
\end{tabular}

In "Values" column, $\mathcal{U}$ refers to a uniform distribution. Dimensions are mass $(M)$, length $(L)$, time $(t)$, and temperature $(\Theta)$

rates with temperature), with $k$ being the constant of Boltzmann and $E$ the activation energy. The allometric constants $a_{y_{\left(T_{0}\right)}}$ and $a_{x_{\left(T_{0}\right)}}$ correspond to the physiological maxima measured at the reference temperature $T_{0}=20^{\circ} \mathrm{C}$. For the sake of simplicity, we assume identical allometric constants for the different species of a given trophic level, and identical activation energy $E=0.62 \mathrm{eV}$ for biological rates of all species (a mean value across many organism groups: Gillooly et al. 2001; Vasseur and McCann 2005), even though we are aware of recent studies documenting differences between species within a given trophic level (Rall et al. 2012). Model 2 will be used for all simulations performed to answer Q2 and Q3. In addition, we use two variants of model 2 (leading to models 3 and 4 hereafter presented) to assess the sensitivity of trait selection to simple ecological assumptions commonly found in previous models. In model 3, we divide the uptake rate by the conversion efficiency, $\varepsilon_{j}$, rather than multiplying the production rate:

$$
\begin{aligned}
& \frac{d R_{i}}{d t}=I-\mathrm{out}_{R} R_{i}-\sum_{j} Y_{j} R_{i} P_{j} / \varepsilon_{j} \\
& \frac{d P_{j}}{d t}=\sum_{i} Y_{j} R_{i} P_{j}-X_{j} P_{j}
\end{aligned}
$$

This way of modeling the energy loss between uptake and biomass production is less intuitive but commonly used in bioenergetic models (e.g., Brose et al. 2006b; Berlow et al. 2009). Both formulations lead to the same effective conversion efficiency since in model 3 the production of one unit of consumer requires $1 / \varepsilon_{j}$ units of the resource, while in model 2 , species produce $\varepsilon_{j}$ unit of biomass per unit of resource consumed. Then this distinction seems trivial, but since we expect the equilibria of model 3 to differ from model 2, this assumption might impact trait selection. In model 4, we consider that species also loose biomass independently from their catabolism at a constant rate out $_{P}$. This encompasses many common processes not explicitly considered here, such as sedimentation in aquatic systems, emigration, or predation by other species. This additional outflow is typically integrated in ecosystem models (DeAngelis and Mulholland 1989). See Table 4 in Online Appendix B for a comparative summary of the models.

Invasion analysis Traits potentially under selection are the conversion efficiency $\varepsilon_{j}$ in all four models, the mortality and the per capita uptake rates $m_{j}$ and $a_{j}$ in model 1 , and the body mass $M_{j}$ in models 2 to 4 . To understand how these traits are selected during the assembly, we study the limiting trait values 
enabling a species to invade an ecosystem where a single species is already established (Fig. 1a). As an example, here we describe the simple case of model 1 and provide the results of the full analysis in the "Results" section. We envision the simplest case where a species $P_{2}$, which is initially rare, tries to colonize an ecosystem occupied by a resident species $P_{1}$ already at equilibrium (Fig. 1a). Both producers consume the same resource $R$. We ask: what conditions on traits ( $a, m$ and $\varepsilon$ ) allow $P_{2}$ to invade the ecosystem? If $P_{1}$ and the resource are at equilibrium, then

$R^{*}=\frac{m_{P_{1}}}{a_{P_{1}} \varepsilon_{P_{1}}}$

$P_{2}$ must have a positive growth rate to invade when at low density (i.e., $P_{2} \ll P_{1}$ ), such that

$\frac{d P_{2}}{d t}=\varepsilon_{P_{2}} a_{P_{2}} R^{*} P_{2}-m_{P_{2}} P_{2}>0$

Replacing [7] into [8] leads to the following invasion condition:

$\frac{m_{P_{2}}}{a_{P_{2}} \varepsilon_{P_{2}}}<\frac{m_{P_{1}}}{a_{P_{1}} \varepsilon_{P_{1}}}$

Since only exploitative competition is at play in this model with a single trophic level, the invasion condition boils down to the $R^{*}$ rule (Tilman 1982): the species persisting is the one whose traits minimize the availability of the resource at equilibrium, $R^{*}$. From inequality [9], the three traits $a, m$, and $\varepsilon$ are under selection in model 1. Exploitative competition will select lower mortality rates $m_{i}$ and greater consumption rates $a_{i}$ and conversion efficiencies $\varepsilon_{i}$. We performed identical analyses on each of the three bioenergetic models (models 2 , 3 , and 4).

Simulations We then investigate the analytical predictions in a multi-species context with simulations of an assembly process (an example is illustrated in Fig. 1d). The ecosystem is initially empty and contains ten different resources, corresponding to different niches for the producers. At each assembly step, we randomly select a species and attempt an invasion. Traits potentially under selection are randomly drawn from uniform distributions (see Table 1), and each species is attributed one to three resources randomly chosen. When a species feeds on more than one resource, we divide the uptake uniformly across the resources with a preference parameter $\omega=1 /$ \# resources to avoid giving a competitive advantage to generalist species. We then rewrite the set of equations describing the ecosystem dynamics including the invader, with an initial small biomass $\left(10^{-4}\right.$ units), and run the numerical integration with the RungeKutta Kash-Carp algorithm (gsl 1.15 library; Galassi et al. 2011) until the equilibrium is reached (i.e., when biomass averaged over 500 integration steps varies less than $10^{-11}$ units).
Species whose biomass falls below a threshold of $10^{-6}$ units are considered extinct. A step consists of a successful invasion, when the invader persists while dynamics are run until the equilibrium. An assembly sequence consists of successive invasions and stops either if a trait no longer varies (less than $5 \%$ among species in the food web), if the invader does not manage to double its initial small density, or after 10,000 unsuccessful invasion trials within a step. In these three cases, equilibrium is considered reached at the scale of assembly. We run 50 simulations per model, and we track how traits deviate from their initial uniform distribution along the assembly. For each of the four models, we compare the trait selection between models and between simulations and analytical predictions.

\section{Q2 Effect of adding a trophic level}

Next, we add herbivores, $H$, to understand if they alter the identity of traits under selection and the direction of the selection. As an example, we show below the equations of model 2 , later used for multispecies simulations ( $Y$ and $X$ described in [7] and [8]):

$$
\begin{aligned}
& \frac{d R_{i}}{d t}=I-\text { out }_{R} R_{i}-\sum_{j} Y_{j} R_{i} P_{j} \\
& \frac{d P_{j}}{d t}=\varepsilon_{j} \sum_{i} Y_{j} R_{i} P_{j}-X_{j} P_{j}-\sum_{k} Y_{k} P_{j} H_{k}
\end{aligned}
$$

$\frac{d H_{k}}{d t}=\varepsilon_{k} \sum_{j} Y_{k} P_{j} H_{k}-X_{k} H_{k}$

As for Q1, we carry on invasion analyses for the four models. We consider invasion of either a producer (Fig. 1b) or an herbivore (Fig. 1c), and we analyze trait selection for both trophic levels. Invasion criteria are derived following the same reasoning than for Q1. We compare analytical predictions with and without herbivores among models 1, 2, and 3 (equilibria of model 4 with an herbivore are not tractable). We then focus on the bioenergetic model 2 for the multi-species simulations. We simulate 200 food web assemblies, either with or without herbivores. Herbivores are allowed to eat all producers whose body mass fall into a certain range of values (Riede et al. 2011; see Fig. 6 in Online Appendix C). The boundaries of the diet are given by the 10 and $90 \%$ quantile regressions on a well-documented linear empirical relationship between prey and predator logarithm of the body mass (Brose et al. 2006a; Gravel et al. 2013). Subsequent simulations follow the same procedure as described for Q1, except that we stop the assembly after 200 steps to have comparable length of assembly with and without herbivores though herbivore 
turnover allows longer assembly processes. We plot the body mass and efficiency distributions of producers and herbivores present in the ecosystem at the end of the assembly, pooling species by type over all simulations, for each simulation set "with" and "without herbivores."

\section{Q3 Effect of temperature}

Ultimately, we investigate analytically how temperature affects the invasion criteria previously established using small modules (for cases described in Fig. 1a-c). We then simulate 200 food web assemblies using model 2 including herbivores at two contrasted temperatures: $T=\left\{0^{\circ} C ; 40^{\circ} C\right\}$. As for Q2, we examine if the deviation of traits from their initial uniform distribution supports our analytical predictions and we compare trait selection at the end of assembly between the two temperature treatments.

\section{Results}

\section{Trait selection and structural sensitivity}

We find that trait selection varies significantly between models in competitive communities (Table 2.1). Because in that situation, selection is driven only by exploitative competition, the outcome depends on the resource equilibrium, $R^{*}$ (see Online Appendix A for equilibria, Table 3.1), and thereby trait selection depends on the traits of the producer. This trait selection, however, varies among models. For model $1, R^{*}$ combines all the traits of the producer $\left(R^{*}=\frac{m_{P}}{a_{P} \varepsilon_{P}}\right)$, namely, the uptake rate, $a_{P}$ the mortality rate, $m_{P}$, and the conversion efficiency, $\varepsilon_{P}$ By contrast, the $R^{*}$ is independent from body mass $M_{P}$ in models 2 $\left(R^{*}=\frac{a_{x}}{a_{y} \varepsilon_{P}}\right)$ and $3\left(R^{*}=\frac{a_{x}}{a_{y}}\right)$. Body mass is under selection only when there are outputs $\mathrm{out}_{P}$ independent from the producer metabolism (i.e., in model 4: $R^{*}=\frac{a_{x}}{a_{y} \varepsilon_{P}}\left(1+\frac{\text { out }_{P}}{a_{x} M_{P}^{-0.25}}\right)$;
Table 2.1). In that case, and under identical output rates, this would favor the species with the lowest body mass. Finally, models 1, 2, and 4 predict that higher conversion efficiencies would be selected by allowing an increase in species resource uptake relatively to output flows (Table 2.1). In model 3, however, $R^{*}$ is determined by traits that we do not investigate in this study (allometric coefficients $a_{x}$ and $a_{y}$ ), and thus, none of the traits allowed to vary ( $M$ or $\varepsilon$ ) are under selection.

The multispecies simulations of competitive communities (Fig. 2) are coherent with the analytical results (Table 2.1). Traits under selection quickly reach their minimum or maximum value in the direction predicted by the invasion analyses, while the other traits remain close to the expectation of the uniform distribution. It is noteworthy that the selection pressure is relaxed when several traits are selected in the same direction (e.g., higher attack rates and conversion efficiencies in model 1; Fig. 2a): the traits remain more variable than in the case where a single trait is under selection (Fig. 2f). We also observe that when no selection operates on the traits (Table 2.1, model 3), the first species arrived preempts the resources and later invaders are not able to grow, which, based on our simulation rules, quickly stops the assembly (model 3, Fig 2c, g).

\section{Trophic complexity}

This variability of trait selection depending on the model (i.e., which traits are selected and in which direction) is mitigated with the addition of herbivores: producers with small body masses outcompete larger resident producers regardless of the model studied (Table 2.2, left column; Online Appendix D for details). Thus, under herbivory, the trait selection of the basal species becomes less sensitive to the model structure. However, herbivore traits are under the same selection pressure, driven by exploitative competition, as what we find for competitive communities, which depends on the model in the same way as discussed in the previous paragraph.

Table 2 Direction of trait selection, as derived from the analysis of small modules (see Fig. 1a-c), for each of the four models (1 to 4 )

\begin{tabular}{|c|c|c|c|}
\hline & Model & Plant trait selection & Herbivore trait selection \\
\hline \multirow[t]{4}{*}{$\begin{array}{l}2.1 \text { Without herbivore } \\
\text { (Fig. 1a) obtained from } R^{*} \text { equilibria (Table } 3 \text { ) }\end{array}$} & Model 1 & $m_{p} \downarrow, \quad a_{p} \uparrow \quad$ et $\quad \varepsilon_{p} \uparrow$ & \\
\hline & Model 2 & $\varepsilon_{P} \uparrow$ & \\
\hline & Model 3 & $\varnothing$ & \\
\hline & Model 4 & $M_{P} \downarrow \varepsilon_{P} \uparrow$ & \\
\hline \multirow{3}{*}{$\begin{array}{l}2.2 \text { With herbivore } \\
\text { (Fig. } 1 \mathrm{~b}, \mathrm{c} \text { ) obtained from invasion analysis for } P^{\prime} \text { 's traits } \\
\quad\left(\text { Table } 5 \text { ) and from } P^{*} \text { for } H^{\prime} \text { 's traits (Table } 3 \text { ) }\right.\end{array}$} & Model 1 & $m_{p} \downarrow, \quad a_{p} \uparrow \quad$ et $\quad \varepsilon_{p} \uparrow$ & $m_{H} \downarrow, \quad a_{H} \uparrow \quad$ et $\quad \varepsilon_{H} \uparrow$ \\
\hline & Model 2 & $M_{P} \downarrow \varepsilon_{p} \uparrow$ & $\varepsilon_{H} \uparrow$ \\
\hline & Model 3 & $M_{P} \downarrow$ & $\varnothing$ \\
\hline
\end{tabular}

Upward arrows indicate selection to greater values of the trait, while downward arrows indicate selection to lower values. Temperature dependence is not considered here ( $T=T_{0}$ in models 2 to 4$)$. Equilibria were not tractable for model 4 when the herbivore was present 


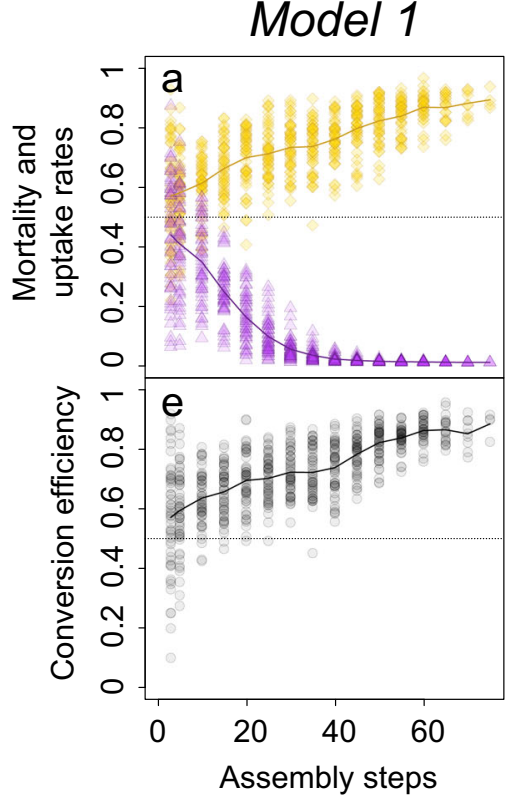

Fig. 2 Variation of producer traits along food web assembly for each of the four models considered. Symbols show the average value of a trait over all the species in a community at a given assembly step (one simulation). a Yellow diamonds and purple triangles refer to uptake and

Simulations of model 2 with herbivores result in a bimodal distribution of the producer body mass at the end of the simulation (Fig. 3d green solid line), while the analytical criteria predict only a selection toward lower values (Table 2.2). Actually, we find a mixture of small primary producers experiencing herbivory (Fig. 3e light green line) and very large or very small primary producers free of herbivory (Fig. 3e, dark green line). The distribution of producers subject to herbivory is clearly biased toward smaller body masses (Fig. 3e, light green line), which is congruent with the analytical predictions. Simulations also confirm the selection of higher conversion efficiencies (Fig. 3f). In addition, they show that when herbivores are present, the strength of selection on conversion efficiency is relaxed: we observe a larger variance of efficiency distributions with than without the presence of herbivores (compare panels $\mathrm{c}$ and $\mathrm{f}$ in Fig. 3).

\section{Temperature}

Analysis on small modules shows two effects of temperature on trait selection, potentially acting in opposite directions: temperature can either directly affect the competition outcome, notably between producers under herbivory (Online Appendix D, Table 5 for the invasion conditions), or reduce the resource availability for herbivores (Online Appendix D, Table 6). Temperature generally does not affect the analytical invasion criteria (Online Appendix D, Table 5); however, in cases of competition between very similar producers, under herbivory, an increase in temperature favors the largest or the less efficient species, thereby decreasing the global selection pressure on traits (Online Appendix D for a detailed analysis). Conversely, a higher temperature also indirectly increases the strength of the selection on herbivore traits by decreasing the density of producers available $\left(\mathrm{P}^{*}\right.$ without the herbivore diminishes: equilibria Table 3.1 and Table 6.1 in Online Appendices $\mathrm{A}$ and $\mathrm{D})$. At high temperatures, the maximal possible efficiency of an herbivore $\left(\varepsilon_{P}=1\right)$ becomes insufficient to enable the herbivores that feed on the smallest and less efficient producers to persist in the system (Fig. 4).

As a result, a larger temperature has a striking impact on the structure of simulated multispecies assemblages (Fig. 5). The proportion of small primary producers rises with temperature, confirming analytical results, while the smallest herbivores cannot maintain themselves and disappear (compare panels $a$ and $d$ in Fig. 5). Thus, increased temperature promotes the emergence of invulnerable small primary producers (Fig. 5e). Moreover, conversion efficiencies of both producers and herbivores are more strongly selected at high temperatures, as shown by the reduced variance in conversion efficiencies (compare panels $\mathrm{c}$ and $\mathrm{f}$ in Fig. 5). Overall, at warmer temperatures, the interactions between residents and invaders during the assembly process select smaller producers and larger herbivores, with higher conversion efficiencies for both. 
Fig. 3 Frequency distributions of the body mass (top and middle panels) and conversion efficiency (bottom panels) of primary producers (solid green lines) and herbivores (dashed red lines) at the end of 200 simulated assemblies of model 2, either without (left panels) or with herbivores (right panels). Middle panels display separately the producers that are fed upon (light green line) or not (dark green lines) by an herbivore in the producer distributions of the top panels. The horizontal dotted line indicates the initial trait distributions. The vertical dotted line indicates the critical body mass above which a producer is too large for herbivores (Online Appendix C Fig. 6). To compare with Fig. 4, temperature is $\mathrm{T}=20^{\circ} \mathrm{C}$
Without herbivores

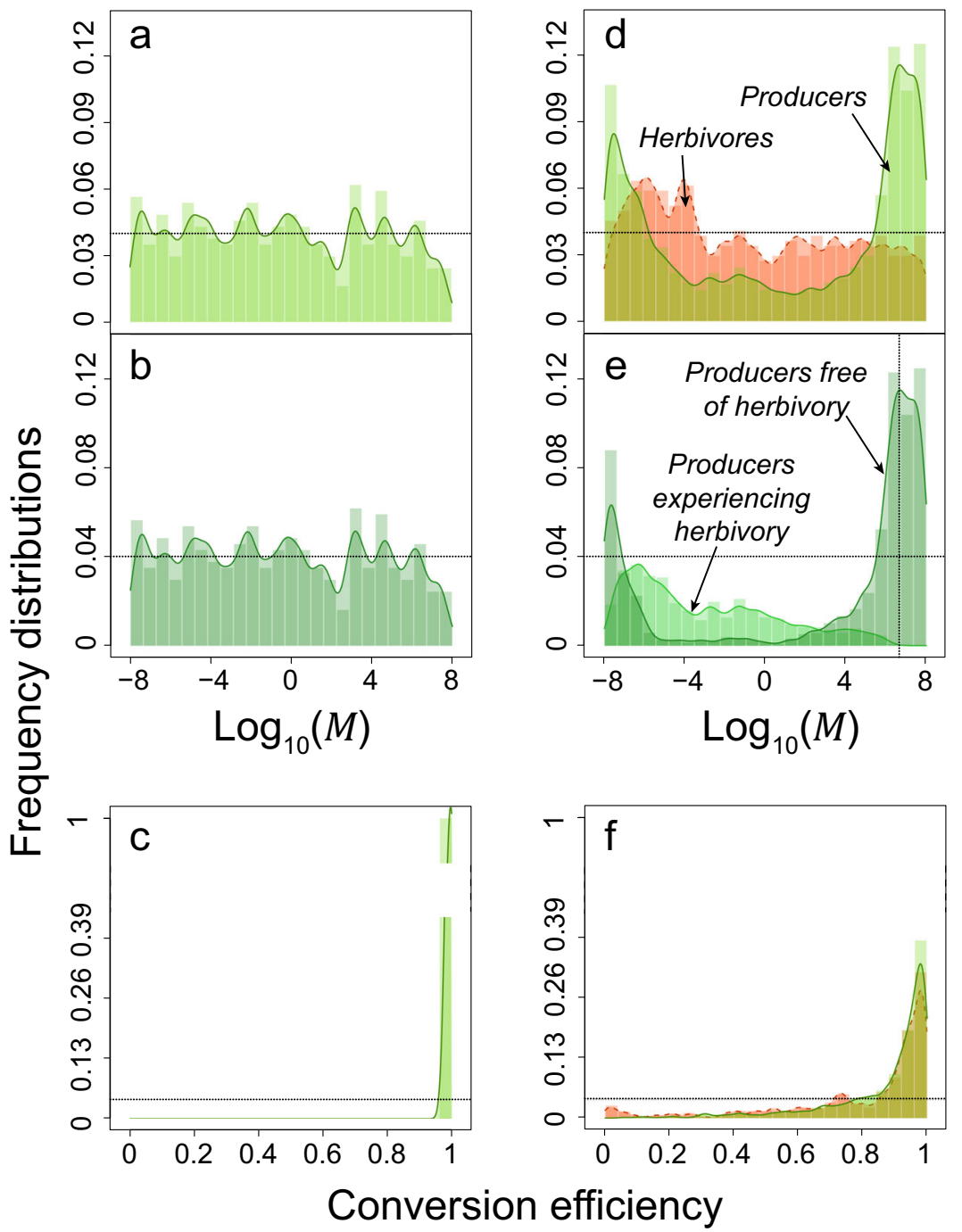

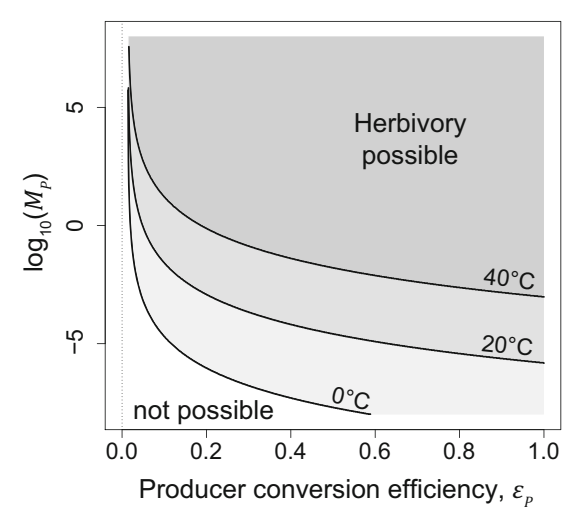

Fig. 4 Isoclines of herbivore critical conversion efficiency: $\varepsilon_{H}{ }^{\text {crit }}=1$, according to the conversion efficiency, $\varepsilon_{P}$ and the log of the body mass, $M_{P}$ of its single prey, and for temperatures 0,20 , and $40{ }^{\circ} \mathrm{C}$. Critical conversion efficiency is the minimal efficiency to insure herbivore persistence. The grey areas show the producer parameter space where an herbivore can settle in the system, i.e., where an herbivore with an efficiency lower than 1 (the maximum) can have sufficient food to persist

\section{Discussion}

Our results show that food web assembly generally promotes the selection of small producers with high conversion efficiency. However, which trait is under selection strongly depends on model structure. Moreover, the presence of a higher trophic level and of temperature dependency modulates the strength of the selection, which in turn shapes the trait distribution emerging from the assembly process.

One first interesting result is the sensitivity of trait selection to the representation of energy flows. What appears trivial at first glance, i.e., different formulations of conversion efficiency (model 2 versus 3 ), strongly affects the equilibria. The conversion efficiency is part of the resource equilibrium, $\mathrm{R}^{*}$, when multiplied with the consumption term (i.e., in model 2), but not when it divides the uptake term (i.e., in model 3). Subsequently, in the former case, successive competition 
Fig. 5 Frequency distributions of the logarithm of body mass (top and middle panels) and conversion efficiency (bottom panels) of primary producers (solid green lines) and herbivores (dashed red lines) at the end of 200 simulated assemblies of model 2, either at temperature $\mathrm{T}=0{ }^{\circ} \mathrm{C}$ (left panels) or $\mathrm{T}=40^{\circ} \mathrm{C}$ (right panels). Middle panels display separately the producers that are fed upon (light green line) or not (dark green lines) by an herbivore in the producer distributions of the top panels. The horizontal dotted line indicates the initial trait distributions. The vertical dotted line indicates the critical body mass above which a producer is too large for herbivores (online Appendix C Fig. 6)

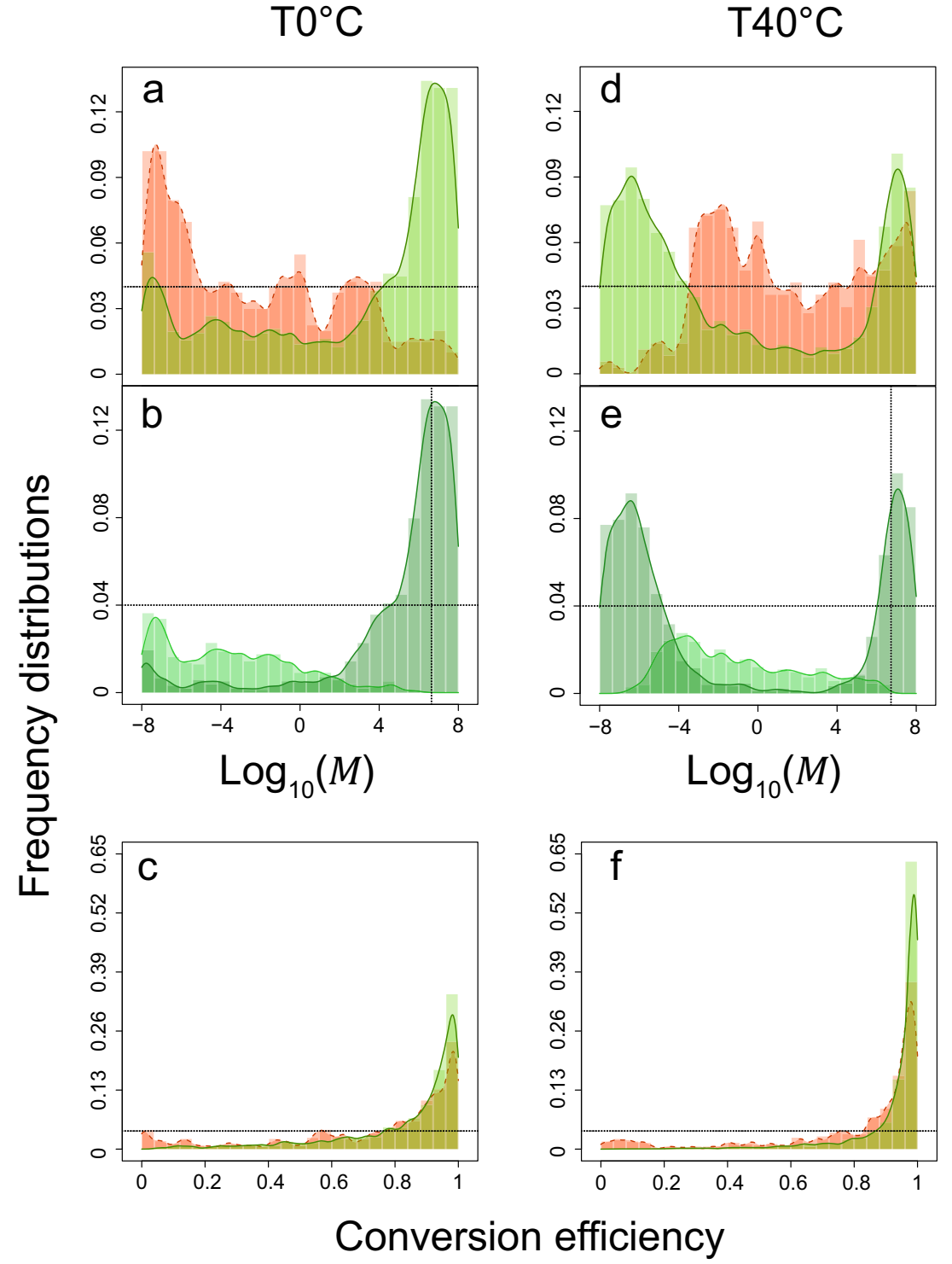

events select more efficient species, whereas in the later invasion success depends neither on conversion efficiency nor on body mass. Species arriving later in model 3 are not able to increase in abundance due to resource preemption. Their low density would inevitably make them subjected to drift (Gravel et al. 2011). The selection therefore becomes neutral, and the trait distribution results only from the order of species arrival. We also find that trait selection varies depending on whether mass-independent losses of biomass are considered or not (i.e., models 4 versus 2, Fig. 2), which determines if smaller body masses are selected or not. This suggests that food web studies performed with bioenergetic models should discuss their results in the light of the selection effects induced by their basic ecological assumptions.

Our results also suggest that trophic complexity tempers the variability of trait selection with model assumptions: in the presence of herbivores, smaller body masses are selected for producers in all bioenergetic models (Table 2.2). The sensitivity to model formulations holds only for the upper trophic level in diverse networks, where only exploitative competition is at work. Simulations also show that selection is weaker for herbivores compared to producers likely due to high herbivore turnover. At a lower trophic level, producers subject to herbivory must be able to stand the highest herbivore density to win the competition (Holt et al. 2001), which implies to be the most productive and, subsequently, to have the smallest body mass. Such selection of small organisms has been demonstrated for instance in benthic communities under increasing fish predation pressure (Blumenshine et al. 2000). Very small sizes of heterotrophic bacteria or pico-plankton have also been hypothesized to act as a refuge against predation (Jumars et al. 1993; Boenigk et al. 2004). Large sizes can constitute a refuge for prey as well (Jürgens and Sala 2000; Smetacek et al. 2004; Klauschies et al. 2012). For instance, 
large herbivores suffer lower predation rates than smaller ones in arctic tundra (Legagneux et al. 2014), and the proportion of large unicellular algae increases after grazing peaks of rotifers in plankton communities (Gosselain et al. 1998).

Our model generates a bimodal distribution of body mass for producers under herbivory that fits both of these size-refuge empirical observations. The selection exerted by resource competition under herbivory produces a group of small producers, and a group of large producers that persist, despite a low productivity, by avoiding herbivory. This size refuge would not appear if we extended the herbivore size range to allow greater sizes with trophic level (Cohen et al. 2003; Woodward et al. 2005). However, food webs may evolve in size clusters with size gaps without consumer species (Loeuille and Loreau 2005), and large size-refuges may emerge in nature from the absence of larger generalist predators for specific prey groups: food web assembly with our bioenergetic model efficiently reproduces how consumer-resource interactions may shape the body mass distributions of producers, as commonly found in plankton communities where two strategies can coexist - grazing avoidance with large sizes and nutrient use improvement with small sizes (Matz and Jürgens 2003; Thingstad et al. 2005).

Our study also shows that trait distributions in food webs crucially depend on temperature. Notably, a warmer temperature leads to an increase in the proportion of small producers in the presence of herbivores. This last result is consistent with what has been found for plankton both experimentally (Peter and Sommer 2012) and in comparative studies (Hilligsøe et al. 2011). Many hypotheses have been proposed in the literature to explain size reduction with temperature (Daufresne et al. 2009; Brose et al. 2012; Hessen et al. 2013). Several of them are physiology-based explanations, including priority to cell division over cell growth under increased temperatures (Van der Have and De Jong 1996), or larger sizes allowed by a better conversion efficiency under colder temperatures (Karl and Fischer 2008), which maximizes the reproductive success accounting for a short reproductive season (Angilletta et al. 2004). We have not implemented ontogenic or plasticity mechanisms in our model, which would produce direct responses of species size to temperature. Nonetheless, we find that temperature also indirectly influences food web size structure, with the modification of resource availability and interaction strength as reported in empirical data (Rall et al. 2010; Forster et al. 2012; Yom-Tov et al. 2006). The gain in metabolic activity with temperature reduces the equilibrium density of primary producers in our simulations, as predicted by the metabolic theory of ecology (Brown et al. 2004; Savage et al. 2004), making resources less available for herbivores. The smallest producers become invulnerable while the smallest herbivores go extinct. Similarly, warming experiments displayed a shift to autotrophdominated structures following the loss of predators and herbivores (Petchey et al. 1999; Pounds et al. 1999). The emergence of small invulnerable primary producers in our food webs increases the strength of exploitative competition among them and among herbivores as well, and leads to a stronger selection pressure toward greater conversion efficiencies. The individual ingestion efficiency (gain by ingestion relative to metabolic losses) of arthropod predators was found experimentally to decrease with warming (Rall et al. 2010). Our model shows that the impact of temperature on food web structure may compensate this possible plastic response on ingestion by a selection for higher conversion efficiencies via the assembly process.

Our study focuses on the mechanisms driving trait selection in food web assembly, assuming several over-simplifications. It would be relevant to relax some of these assumptions by, for instance, using parameter values for other groups than invertebrate herbivores, or implementing interspecific variability in the dependence of biological rates to body mass and temperature, notably using species - and rate - specific activation energies (Price et al. 2012; Humphries and McCann 2014). Moreover, different biological rates potentially respond differently to temperature (Gilbert et al. 2014). For instance, warming may increase feeding rate but decrease efficiency, leading to more stable prey-predator dynamics (Vucic-Pestic et al. 2011). We expect that introducing interspecific variability in allometric constants should reduce the selection pressure exerted on efficiency and body mass by adding other dimensions to selection.

Two other important features must be addressed in future work: greater trophic complexity and non-linear dynamics. Trophic complexity includes adding carnivores and top carnivores, and allowing for omnivory, which is common in natural systems (Polis 1991; Diehl 1993) and contributes actively to ecosystem stability (Holyoak and Sachdev 1998). We expect trophic complexity, especially predation (Chase et al. 2009), and the potential associated decrease in interaction strength (Berlow 1999) to reduce the intensity of trait selection. Linear dynamics may provide an acceptable approximation of trait selection in simple systems, such as plankton, but the study of more diverse webs requires integrating non-linear functional responses, which induces additional coexistence mechanisms (Huisman and Weissing 1999; Drossel et al. 2004; Gravel et al. 2011). The extent to which non-linear dynamics modify trait selection, and temperature effects on trophic structure, remains an open question.

\section{Conclusion}

Our integration of consumer-resource theory with food web assembly using bioenergetic models provides theoretical bases to understand trait selection and how the assembly process shapes body mass distributions in food webs. Further investigations should also consider how assembly features, such as faster immigration rates, might affect trait 
selection by relaxing competitive exclusion. Ultimately, variations in trait distributions are likely to affect ecosystem properties (Virgo et al. 2006). Since body mass is negatively correlated with productivity, ecosystem productivity and biomass production may closely vary with body mass during the assembly process (Schramski et al. 2015). Similarly, the heavy structural changes that we observe in response to temperature variation should strongly feed back on ecosystem functioning. Finally, our study shows that bioenergetic assembly models are exciting tools to revisit ecosystem development understanding with mechanisms bridging traits and ecosystem functioning (Loreau 2010).

Acknowledgments We thank Tanguy Daufresne, Ulrich Brose, Daniel Stouffer, and Barbara Drossel for early discussions and helpful suggestions. We also thank James Caveen, Rémy Dernat, and Khali Belkhir for technical assistance. The simulations largely benefited from the computing clusters of Université du Québec à Rimouski and from the Montpellier Bioinformatics Biodiversity platform (funded by the LabEx CeMEB). I.G. thanks the Frontenac program (Fonds de recherche du Québec-Nature et Technologies, and French consulate at Québec) for their financial support. This is ISEM publication number ISEM 2016-061.

\section{References}

Angilletta MJ, Steury TD, Sears MW (2004) Temperature, growth rate, and body size in ectotherms: fitting pieces of a life-history puzzle. Integr Comp Biol 44:498-509

Bascompte J, Stouffer DB (2009) The assembly and disassembly of ecological networks. Philos Trans R Soc Lond B Biol Sci 364:1781-7. doi:10.1098/rstb.2008.0226

Berlow EL (1999) Strong effects of weak interactions in ecological communities. Nature 398:330-334

Berlow EL, Dunne JA, Martinez ND et al (2009) Simple prediction of interaction strengths in complex food webs. Proc Natl Acad Sci 106: 187-191

Blumenshine SC, Lodge DM, Hodgson JR (2000) Gradient of fish predation alters body size distributions in Benthos. Ecology 81:374-386

Boenigk J, Stadler P, Wiedlroither A, Hahn MW (2004) Strain-specific differences in the grazing sensitivities of closely related ultramicrobacteria affiliated with the Polynucleobacter cluster. Appl Environ Microbiol 70:5787-5793. doi:10.1128/AEM.70. 10.5787

Brose U, Jonsson T, Berlow EL et al (2006a) Consumer-resource bodysize relationships in natural food webs. Ecology 87:2411-2417

Brose U, Williams RJ, Martinez ND (2006b) Allometric scaling enhances stability in complex food webs. Ecol Lett 9:1228-1236. doi:10. 1111/j.1461-0248.2006.00978.x

Brose U, Dunne JA, Montoya JM et al (2012) Climate change in sizestructured ecosystems. Philos Trans R Soc Lond B Biol Sci 367: 2903-12. doi:10.1098/rstb.2012.0232

Brown JH, Gillooly JF, Allen AP et al (2004) Toward a metabolic theory of ecology. Ecology 85:1771-1789

Capitán JA, Cuesta JA, Bascompte J (2011) Species assembly in model ecosystems, I: analysis of the population model and the invasion dynamics. J Theor Biol 269:330-43. doi:10.1016/j.jtbi.2010.09.032

Case TJ (1990) Invasion resistance arises in strongly interacting species-rich model competition communities. Proc Natl Acad Sci 87:9610-9614
Case TJ (1991) Invasion resistance, species buil-up and community collapse in metapopulation models with interspecies competition. Biol J Linn Soc 42:239-266

Chase JM, Leibold MA (2003) Ecological niches-linking classical and contemporary approaches. The University of Chicago press, Chicago and London

Chase JM, Abrams PA, Grover JP et al (2002) The interaction between predation and competition: a review and synthesis. Ecol Lett 5:302315. doi:10.1046/j.1461-0248.2002.00315.x

Chase JM, Biro EG, Ryberg WA, Smith KG (2009) Predators temper the relative importance of stochastic processes in the assembly of prey metacommunities. Ecol Lett 12:1210-8. doi:10.1111/j.1461-0248. 2009.01362.x

Chesson P, Kuang JJ (2008) The interaction between predation and competition. Nature 456:235-8. doi:10.1038/nature07248

Cohen JE, Jonsson T, Carpenter SR (2003) Ecological community description using the food web, species abundance, and body size. PNAS 100:1781-1786

Daufresne M, Lengfellner K, Sommer U (2009) Global warming benefits the small in aquatic ecosystems. Proc Natl Acad Sci 106:12788-12793

DeAngelis D, Mulholland P (1989) Nutrient dynamics and food web stability. Annu Rev Ecol Syst 20:71-95

Diehl S (1993) Relative consumer sizes and the strength of direct and indirect interactions in omnivorous interactions feeding relationships. Oikos 68:151-157

Drossel B, McKane AJ, Quince C (2004) The impact of nonlinear functional responses on the long-term evolution of food web structure. J Theor Biol 229:539-548

Dunne JA, Williams RJ (2009) Cascading extinctions and community collapse in model food webs. Philos Trans R Soc Lond B Biol Sci 364:1711-23. doi:10.1098/rstb.2008.0219

Dunne JA, Williams RJ, Martinez ND (2002) Network structure and biodiversity loss in food webs: robustness increases with connectance. Ecol Lett 5:558-567. doi:10.1046/j.1461-0248.2002. 00354.x

Forster J, Hirst AG, Atkinson D (2012) Warming-induced reductions in body size are greater in aquatic than terrestrial species. Proc Natl Acad Sci U S A 109:19310-4. doi:10.1073/pnas.1210460109

Fowler MS (2010) Extinction cascades and the distribution of species interactions. Oikos 119:864-873. doi:10.1111/j.1600-0706.2009. 17817.x

Fukami T (2005) Integrating internal and external dispersal in metacommunity assembly: preliminary theoretical analyses. Ecol Res 20:623-631. doi:10.1007/s11284-005-0092-3

Galassi M, Davies J, Theiler J, et al. (2011) GNU Scientific Library Reference Manual, 3rd edn

Gause GF (1934) The struggle for existence. Wilkins \& Williams, Baltimore

Gilbert B, Tunney TD, McCann KS et al (2014) A bioenergetic framework for the temperature dependence of trophic interactions. Ecol Lett 17:902-14. doi:10.1111/ele.12307

Gillooly JF, Brown JH, West GB et al (2001) Effects of size and temperature on metabolic rate. Science 293:2248-51. doi:10.1126/science. 1061967

Gosselain V, Viroux L, Descy JP (1998) Can a community of small-bodied grazers control phytoplankton in rivers ? Freshw Biol 39:9-24

Götzenberger L, de Bello F, Bråthen KA et al (2012) Ecological assembly rules in plant communities - approaches, patterns and prospects. Biol Rev Camb Philos Soc 87:111-27. doi:10.1111/j.1469-185X. 2011.00187.x

Gravel D, Guichard F, Hochberg ME (2011) Species coexistence in a variable world. Ecol Lett 14:828-39. doi:10.1111/j.1461-0248. 2011.01643.x

Gravel D, Poisot T, Albouy C et al (2013) Inferring food web structure from predator-prey body size relationships. Methods Ecol Evol 4: 1083-1090. doi:10.1111/2041-210X.12103 
Gross K, Cardinale BJ (2005) The functional consequences of random vs. ordered species extinctions. Ecol Lett 8:409-418. doi:10.1111/j. 1461-0248.2005.00733.x

Hardin G (1960) The competitive exclusion principle. Science 131:12921297

Hessen DO, Daufresne M, Leinaas HP (2013) Temperature-size relations from the cellular-genomic perspective. Biol Rev Camb Philos Soc 88:476-89. doi:10.1111/brv.12006

Hilligsøe KM, Richardson K, Bendtsen J et al (2011) Linking phytoplankton community size composition with temperature, plankton food web structure and sea-air CO2 flux. Deep Sea Res Part I Oceanogr Res Pap 58:826-838. doi:10.1016/j.dsr.2011.06.004

Holt RD, Lawton JH (1994) The ecological consequences of shared natural enemies. Annu Rev Ecol Syst 25:495-520

Holt RD, Grover JP, Tilman D (1994) Simple rules for interspecific dominance in systems with exploitative and apparent competition. Am Nat 144:741-771

Holt RD, Grover JP, Tilman D (2001) Simple rules for interspecific dominance in systems with exploitative and apparent competition. Am Nat 144:741-771

Holyoak M, Sachdev S (1998) Omnivory and the stability of simple food webs. Oecologia 117:413-419

Huisman J, Weissing FJ (1999) Biodiversity of plankton by species oscillations and chaos. Nature 402:407-410

Humphries MM, McCann KS (2014) Metabolic ecology. J Anim Ecol 83: 7-19. doi:10.1111/1365-2656.12124

Jumars PA, Deming JW, Hill PS et al (1993) Physical constraints on marine osmotrophy in an optimal foraging context. Aquat Microb Food Webs 7:121-159

Jürgens K, Sala MM (2000) Predation-mediated shifts in size distribution of microbial biomass and activity during detritus decomposition. Oikos 91:29-40

Karl I, Fischer K (2008) Why get big in the cold? Towards a solution to a life-history puzzle. Oecologia 155:215-25. doi:10.1007/s00442007-0902-0

Klauschies T, Bauer B, Aberle-Malzahn N et al (2012) Climate change effects on phytoplankton depend on cell size and food web structure. Mar Biol 159:2455-2478. doi:10.1007/s00227-012-1904-y

Law R, Morton RD (1993) Alternative permanent states of ecological communities. Ecology 74:1347-1361

Law R, Morton RD (1996) Permanence and the assembly of ecological communities. Ecology 77:762-775

Legagneux P, Gauthier G, Lecomte N et al (2014) Arctic ecosystem structure and functioning shaped by climate and herbivore body size. Nat Clim Chang 4:379-383. doi:10.1038/NCLIMATE2168

Lockwood JL, Powell RD, Nott PM, Pimm SL (1997) Assembling ecological communities in time and space. Oikos 80:549-553. doi: $10.2307 / 3546628$

Loeuille N, Loreau M (2005) Evolutionary emergence of size-structured food webs. Proc Natl Acad Sci 102:5761-5766

Loreau M (2010) Linking biodiversity and ecosystems: towards a unifying ecological theory. Philos Trans R Soc Lond B Biol Sci 365:4960. doi:10.1098/rstb.2009.0155

Louette G, De Meester L (2007) Predation and priority effects in experimental zooplankton communities. Oikos 116:419-426. doi:10.1111/j.2006.0030-1299.15381.x

Luh H-K, Pimm SL (1993) The assembly of ecological communities: a minimalist approach. J Anim Ecol 62:749-765

Martin LM, Wilsey BJ (2012) Assembly history alters alpha and beta diversity, exotic-native proportions and functioning of restored prairie plant communities. J Appl Ecol 49:1436-1445. doi:10.1111/j. 1365-2664.2012.02202.x

Matz C, Jürgens K (2003) Interaction of nutrient limitation and protozoan grazing determines the phenotypic structure of a bacterial community. Microb Ecol 45:384-98. doi:10.1007/s00248-003-2000-0
Miller TE, Burns JH, Munguia P et al (2005) A critical review of twenty years' use of the resource-ratio theory. Am Nat 165:439-48. doi: $10.1086 / 428681$

Montoya JM, Pimm SL, Solé RV (2006) Ecological networks and their fragility. Nature 442:259-64. doi:10.1038/nature04927

Morton RD, Law R (1997) Regional species pools and the assembly of local ecological communities. J Theor Biol 321-331

Murdoch WW, Briggs CJ, Nisbet RM (2003) Consumer-resource dynamics. 462

Olito C, Fukami T (2009) Long-term effects of predator arrival timing on prey community succession. Am Nat 173:354-62. doi:10.1086/ 596538

Petchey OL, McPhearson PT, Casey TM, Morin PJ (1999) Environmental warming alters food-web structure and ecosystem function. Nature 402:69-72

Petchey OL, Beckerman AP, Riede JO, Warren PH (2008) Size, foraging, and food web structure. Proc Natl Acad Sci U S A 105:4191-6. doi:10.1073/pnas.0710672105

Peter KH, Sommer U (2012) Phytoplankton cell size: intra- and interspecific effects of warming and grazing. PLoS ONE. doi:10.1371/ journal.pone. 0049632

Polis GA (1991) Complex trophic interactions in deserts: an empirical critique of food-web theory. Am Nat 138:123-155

Post WM, Pimm SL (1983) Community assembly and food web stability. Math Biosci 64:169-192

Pounds JA, Fogden MPL, Campbell JH (1999) Biological response to climate change on a tropical mountain. Nature 398:611-615

Price CA, Weitz JS, Savage VM et al (2012) Testing the metabolic theory of ecology. Ecol Lett 15:1465-74. doi:10.1111/j.1461-0248.2012. 01860.x

Rall BC, Vucic-Pestic O, Ehnes RB et al (2010) Temperature, predatorprey interaction strength and population stability. Glob Chang Biol 16:2145-2157. doi:10.1111/j.1365-2486.2009.02124.x

Rall BC, Brose U, Hartvig M et al (2012) Universal temperature and body-mass scaling of feeding rates. Philos Trans R Soc Lond B Biol Sci 367:2923-34. doi:10.1098/rstb.2012.0242

Riede JO, Brose U, Ebenman B et al (2011) Stepping in Elton's footprints: a general scaling model for body masses and trophic levels across ecosystems. Ecol Lett 14:169-178. doi:10.1111/j.1461-0248. 2010.01568.x

Savage VM, Gillooly JF, Brown JH, Charnov EL (2004) Effects of body size and temperature on population growth. Am Nat 163:429-41. doi: $10.1086 / 381872$

Schmitz OJ, Hambäck PA, Beckerman AP (2000) Trophic cascades in terrestrial systems: a review of the effects of carnivore removals on plants. Am Nat 155:141-153. doi:10.1086/303311

Schramski JR, Dell AI, Grady JM, et al. (2015) Metabolic theory predicts whole-ecosystem properties. 112:2617-2622. doi: 10.1073/pnas. 1423502112

Schreiber SJ, Rittenhouse S (2004) From simple rules to cycling in community assembly. Oikos 2:349-358

Smetacek V, Assmy P, Henjes J (2004) The role of grazing in structuring Southern Ocean pelagic ecosystems and biogeochemical cycles. Antarct Sci 16:541-558. doi:10.1017/S0954102004002317

Solé RV, Montoya JM (2001) Complexity and fragility in ecological networks. Proc Biol Sci 268:2039-45. doi:10.1098/rspb.2001.1767

Thébault E, Loreau M (2003) Food-web constraints on biodiversity-ecosystem functioning relationships. Proc Natl Acad Sci 100:14949-14954

Thébault E, Loreau M (2006) The relationship between biodiversity and ecosystem functioning in food webs. Ecol Res 21:17-25. doi:10.1007/s11284-005-0127-9

Thingstad TF, Øvreås L, Egge JK et al (2005) Use of non-limiting substrates to increase size; a generic strategy to simultaneously optimize uptake and minimize predation in pelagic osmotrophs? Ecol Lett 8: 675-682. doi:10.1111/j.1461-0248.2005.00768.x 
Tilman D (1977) Resource competition between plankton algae: an experimental and theoretical approach. Ecology 58:338-348

Tilman D (1980) Resources: a graphical-mechanistic approach to competition and predation. Am Nat 116:362-393

Tilman D (1982) Resource competition and community structure. Princeton Monographs in Population Biology 17. Princeton University Press, Princeton

Tilman D, Mattson M, Langer S (1981) Competition and nutrient kinetics along a temperature gradient: an experimental test of a mechanistic approach to niche theory. Limnol Oceanogr 26:1020-1033

Van der Have TM, De Jong G (1996) Adult size in ectotherms: temperature effects on growth and differentiation. J Theor Biol 183:329-340

Vasseur DA, McCann KS (2005) A mechanistic approach for modeling temperature-dependent consumer-resource dynamics. Am Nat 166: 184-98. doi:10.1086/431285
Virgo N, Law R, Emmerson M (2006) Sequentially assembled food webs and extremum principles in ecosystem ecology. J Anim Ecol 75: 377-86. doi:10.1111/j.1365-2656.2006.01058.x

Vucic-Pestic O, Ehnes RB, Rall BC, Brose U (2011) Warming up the system: higher predator feeding rates but lower energetic efficiencies. Glob Chang Biol 17:1301-1310. doi:10.1111/j.1365-2486. 2010.02329.x

Woodward G, Ebenman B, Emmerson M et al (2005) Body size in ecological networks. Trends Ecol Evol 20:402-9. doi:10.1016/j.tree. 2005.04.005

Yodzis P, Innes S (1992) Body size and consumer-resource dynamics. Am Nat 139:1151-1175

Yom-Tov Y, Heggberget TM, Wiig O, Yom-Tov S (2006) Body size changes among otters, Lutra lutra, in Norway: the possible effects of food availability and global warming. Oecologia 150:155-60. doi:10.1007/s00442-006-0499-8 